\title{
Chemiluminescent Detection of Sequential DNA Hybridizations to High-Density, Filter-Arrayed cDNA Libraries: A Subtraction Method for Novel Gene Discovery
}

BioTechniques 27:146-152 (July 1999)

D. Guiliano, M. Ganatra ${ }^{1}$, J. Ware $^{1}$, J. Parrot ${ }^{1}$, J. Daub, L. Moran $^{1}$, H. Brennecke ${ }^{1}$, J.M. Foster $^{1}$, T. Supali ${ }^{2}$, M. Blaxter, A.L. Scott ${ }^{3}$, S.A. Williams ${ }^{4}$ and B.E. Slatko ${ }^{1}$

University of Edinburgh, Edinburgh, Scotland, UK, ${ }^{1}$ New England Biolabs, Beverly, MA, USA, ${ }^{2}$ University of Jakarta, Jakarta, Indonesia, ${ }^{3}$ Johns Hopkins School of Hygiene, Baltimore, MD and ${ }^{4}$ Smith College, Northampton, MA, USA

\footnotetext{
ABSTRACT

A chemiluminescent approach for sequential DNA hybridizations to high-density filter arrays of cDNAs, using a biotinbased random priming method followed by a streptavidin/alkaline phosphatase/CDPStar ${ }^{\mathrm{TM}}$ detection protocol, is presented. The method has been applied to the Brugia malayi genome project, wherein cDNA libraries, cosmid and bacterial artificial chromosome (BAC) libraries have been gridded at high density onto nylon filters for subsequent analysis by hybridization. Individual probes and pools of rRNA probes, ribosomal protein probes and expressed sequence tag probes show correct specificity and high signal-to-noise ratios even after ten rounds of hybridization, detection, stripping of the probes from the membranes and
}

rehybridization with additional probe sets. This approach provides a subtraction method that leads to a reduction in redundant DNA sequencing, thus increasing the rate of novel gene discovery. The method is also applicable for detecting target sequences, which are present in one or only a few copies per cell; it has proven useful for physical mapping of BAC and cosmid highdensity filter arrays, wherein multiple probes have been hybridized at one time (multiplexed) and subsequently "deplexed" into individual components for specific probe localizations.

\section{INTRODUCTION}

The hybridization of nucleic acid probes to identify complementary sequences in complex mixtures of DNA immobilized on membranes forms the basis of many techniques used to analyze gene structure and function $(2,3,16)$. These procedures include the screening of coding DNA (cDNA) and genomic DNA (gDNA) libraries, northern and Southern blot hybridization and hybridization selection and subtraction. The development of chemiluminescent hybridization techniques, in combination with multiplexing/"deplexing" protocols, provides a rapid method that is useful for positional mapping to cDNA, cosmid or bacterial artificial chromosome (BAC) high-density filter arrays and provides a method useful for multiplexed subtraction hybridization. As described below, these procedures are being applied to the analysis of the genome of Brugia malayi, a parasitic nematode that is the causative agent for lymphatic filariasis.

Filariasis affects over 100 million people worldwide, with one billion people at risk from infection from three main species of filarial nematodes (13). Wuchereria bancrofti and B. malayi (and a minor related species $B$. timori) are responsible for lymphatic filariasis, wherein male and female adult worms live in the lymphatic system and cause blockage, leading to the characteristic late-stage symptoms of testicular hydrocoele, elephantiasis and lymphodema. Infective larvae are transmitted to humans by mosquito vectors. The microfilariae migrate to the lymphatics, mature into adult male and female parasites and mate; the females subsequently shed thousands of immature larvae (microfilariae). When a mosquito takes a blood meal, microfilariae are ingested, and the life cycle is reinitiated.

In 1994, the Filarial Genome Network was formed, sponsored by the World Health Organization (WHO) and the UNDP/World Bank/WHO Special Programme for Research and Training in Tropical Diseases (TDR). B. malayi was chosen as a representative candidate for analysis, and cDNA libraries were constructed from seven parasite developmental stages for use as templates to partially sequence expressed genes at random (expressed sequence tags [ESTs]) $(4,6,7,18)$. The information obtained in "one-pass" sequencing is used to query the existing nucleic acid and protein databases to rapidly 
identify novel and previously identified sequences [Basic Local Alignment Search Tool (BLAST) analysis $(1,5,7)]$. Currently, over 16000 ESTs have been deposited in the National Center for Biotechnology Information (NCBI) dbEST database, and methods of "subtraction" are now being applied to reduce redundancy (the probability of randomly resequencing the same EST to increase the frequency of novel gene discovery). Included among these methods are the construction of "subtracted" cDNA libraries (L. Saunders and S. Williams, unpublished). Here, we describe the use of iterative, pooled probe hybridizations to high-density filters to identify previously sequenced ESTs. We also show that cDNAs are useful as templates for chemiluminescent probes to identify, and thus map, overlapping genomic DNA in high-density gridded BAC and cosmid libraries.

\section{MATERIALS AND METHODS}

\section{Construction and DNA Sequencing of the cDNA Libraries}

The construction of cDNA libraries from various life-cycle stages of $B$. malayi has been previously described (7; see also http://www.neb.com/fgn/ filgen1.html). Individual phage were isolated from the B. malayi cDNA libraries made in Lambda ZAP ${ }^{\circledR}$ Express (Stratagene, La Jolla, CA, USA). cDNA inserts from these randomly isolated phage were polymerase chain reaction (PCR)-amplified using AmpliTaq Gold ${ }^{\mathrm{TM}}$ DNA Polymerase (PE Biosystems, Foster City, CA, USA) and flanking T3 and T7 promoter primers, purified by QIAquick ${ }^{\mathrm{TM}}$ (Qiagen, Valencia, CA, USA) or Amicon ${ }^{\circledR}$ Centricon ${ }^{\circledR}-100$ (Millipore, Bedford, MA, USA) and DNA-sequenced using a Model 377 Automated DNA Sequencer with the vector-based SK sequencing primer and DyeDeoxy ${ }^{\mathrm{TM}}$ terminator chemistry (PE Biosystems). Sequence identification was determined by submission of the edited (removal of vector) sequence to GenBank $^{\circledR}$ using BLAST search tools (1) by an automated UNIX ${ }^{\circledR}$-based submission computer program (D. Guiliano, C. Lin and S. Kumar, unpublished). PCR products of interest were selected for use as probes in screening the filters.

\section{High-Density Filters}

Several cDNA libraries were gridded at high density, 18000 clones per $22 \times$ 22-cm filter, with a Genetix ${ }^{\circledR}$ Robot (Genome Systems, St. Louis, MO, USA) onto Hybond ${ }^{\circledR}-\mathrm{N}^{+}$nylon membranes (Amersham Pharmacia Biotech, Piscataway, NJ, USA). Insert-bearing pBluescript $^{\circledR}$ plasmids were mass-excised from the Lambda Zap Express phage vectors following recommended protocols (Stratagene). Subsequent transformants were individually grown in 384-well microtrays and robotically gridded onto the nylon membrane filters at high density. Cosmid (average insert size $30 \mathrm{~kb}$ ) and BAC (average insert size of $70 \mathrm{~kb}$ ) libraries have also been constructed (References 14 and 15; J. Foster, D. Guiliano and B. Slatko, unpublished). Each clone was "double gridded", by the robot, in a known pattern on the high-density filters for more precise confidence of positive hybridizations (see Results). Each filter doublespot position and orientation thus corresponds to a known physical address in a master set of 384-well microplates, in which each original clone resides.

\section{Chemiluminescent Probe Generation}

Biotinylated probes were made with the NEBlot ${ }^{\circledR}$ Phototope $^{\circledR}$ Random Priming Labeling Kit (New England Biolabs, Beverly, MA, USA) and detected with the Phototope ${ }^{\circledR}{ }_{-}$Star Chemiluminescent Detection Kit (alkaline phosphatase/streptavidin/CDP-Star ${ }^{\circledR}$; New England Biolabs). For subtraction methods, probes were made from templates that were found to be in high abundance in the sequenced data set. PCR was performed from the appropriate lambda Zap phage template stock, using vector-based $\mathrm{T} 3$ and $\mathrm{T} 7$ promotor primers and AmpliTaq DNA Polymerase. Each PCR product was restriction endonuclease-digested with EcoRI and XhoI (New England Biolabs) to liberate the insert DNA from the cloning vector and purified from the small vector ends by Amicon Centricon-100 filtration or by QIAquick chromatography. Quan- titation of the product by agarose gel electrophoresis both after PCR and after purification, ensured the correct amount of PCR product for labeling efficiency.

Each purified PCR product was biotin-labeled to high specific activity following the NEBlot Phototope Random Priming Kit protocol using $50-100 \mathrm{ng}$ of PCR product at $37^{\circ} \mathrm{C}$ for 6-10 h. Probe quantity and quality were determined by dot blot analysis of 10 -fold serial dilutions. Probe pools were made from individually labeled and subsequently combined reactions.

\section{Hybridization}

cDNA filters prepared from the $B$. malayi microfilaria (Mf) library and BAC filters containing genomic Brugia DNA were hybridized with biotinylated probes, as follows: each membrane was placed in a hybridization bag (Product No. XQ100B; Tropix, Bedford, MA, USA) and thoroughly wet with $6 \times$ SSC $(20 \times \mathrm{SSC}=3 \mathrm{M} \mathrm{NaCl}, 0.3 \mathrm{M} \mathrm{NaCi}-$ trate, $\mathrm{pH}$ 7.0), followed by prehybridization with $6 \times \mathrm{SSC}, 5 \times$ Denhardt's reagent $\left(50 \times=10 \mathrm{~g} \mathrm{Ficoll}^{\circledR}-400,50 \mathrm{~g}\right.$ polyvinylpyrrolidone, $0.5 \mathrm{~g}$ bovine serum albumin in $500 \mathrm{~mL}$ water), $0.5 \%$ sodium dodecyl sulfate (SDS) and 100 $\mu \mathrm{g} / \mathrm{mL}$ denatured salmon sperm DNA $\left(0.1 \mathrm{~mL}\right.$ of solution per $\mathrm{cm}^{2}$ membrane) for $1 \mathrm{~h}$ at $60^{\circ} \mathrm{C}$. Five to seven microliters of each biotinylated probe were denatured in boiling water for 5 min, chilled on ice for $5 \mathrm{~min}$, centrifuged briefly and then added to the prehybridization solution (the remainder of the probe was used for the "deplexing" procedure described below). Hybridization of the probe was allowed to proceed overnight at $60^{\circ} \mathrm{C}$, with gentle rocking in a hybridization oven (Bokel Industries, American Bioanalytical, Natick, MA, USA).

After hybridization, the membrane was removed from the bag, washed twice in $2 \times \mathrm{SSC}, 0.1 \% \mathrm{SDS}$ at room temperature (RT) for $5 \mathrm{~min}$ each and then washed twice in $0.1 \times \mathrm{SSC}, 0.1 \%$ SDS at $60^{\circ} \mathrm{C}$ for $15 \mathrm{~min}$ each. The washed membrane was placed in a new hybridization bag for subsequent chemiluminescent detection and sealed on three sides, and on the fourth side a small spout was made to add and remove the detection reagents. 


\section{Chemiluminescent Detection}

Phototope-Star detection was carried out (12) using a dialysis clip (Spectra/ Pore ${ }^{\circledR}$ Closures, $144 \mathrm{~mm}$; American Bioanalytical) to seal and unseal the open spout of a hybridization bag between solution removals and additions. Streptavidin, biotinylated alkaline phosphatase and CDP-Star reagents were sequentially added and removed from the bags, with wash steps in between each addition to remove excess reagent. At each step, the bag was rocked for $5 \mathrm{~min}$ at RT with moderate agitation on a shaking rocker (Red Rotor ${ }^{\mathrm{TM}}$; Hoeffer Pharmacia Biotech, San Francisco, CA, USA). After draining the final detection reagent, the membrane was sealed in the bag and exposed to Kodak X-Omat AR (XAR) or Kodak BioMax X-ray film (Scientific Imaging Systems, [Eastman Kodak], New Haven, CT, USA) for 1-2 min, before the film was developed in a Kodak M35A Automated Developing Processor.

Following detection with one probe or probe set, the membranes were stripped and washed to remove the probe. The membranes were rinsed in Milli ${ }^{\circledR}$-Q water (Millipore), incubated in $0.4 \mathrm{~N} \mathrm{NaOH}, 0.1 \% \mathrm{SDS}$ at $90^{\circ} \mathrm{C}$ for $30 \mathrm{~min}$ and then rinsed in $0.2 \mathrm{M}$ Tris$\mathrm{HCI}, 0.1 \times \mathrm{SSC}$ for $30 \mathrm{~min}$ at $25^{\circ} \mathrm{C}$. Membranes were then stored in sealed hybridization bags at $-20^{\circ} \mathrm{C}$. As many as ten strippings and rehybridizations have been performed without loss of hybridization specificity or efficacy.

\section{"Deplexing" the Positive Hybridizations}

After hybridization with pools of probes, positives were identified, and the corresponding clones were selected and grown overnight at $37^{\circ} \mathrm{C}$ on LB agar plus chloramphenicol $(12.5 \mu \mathrm{g} /$ $\mathrm{mL}$ ) plates. From each plate, a single colony was used to begin an overnight $100-\mu \mathrm{L}$ culture in a $96-$ well microtray. After overnight growth, each clone was replica-plated onto a Hybond- $\mathrm{N}^{+}$membrane using a 96-pin "hedgehog" device (V\&P Scientific, San Diego, CA, USA). Multiple copies of the replicaplated membranes were created so that each could be probed with one individual probe from the pooled probe set.
Membranes were placed on LB agar plus chloramphenicol $(12.5 \mu \mathrm{g} / \mathrm{mL})$ plates and grown overnight at $37^{\circ} \mathrm{C}$, colony-side-up. After overnight growth, the membranes were sequentially transferred, colony-side-up, to filter papers saturated with $10 \%$ SDS (3 min for lysis), $0.5 \mathrm{M} \mathrm{NaOH}, 1.5 \mathrm{M} \mathrm{NaCl}$ (5 minutes for denaturation), $0.5 \mathrm{M}$ Tris-base, $\mathrm{pH} 7.0,1.5 \mathrm{M} \mathrm{NaCl}$ (5 min for neutralization) and $2 \times \mathrm{SSC}$ for $5 \mathrm{~min}$. Membranes were baked at $80^{\circ} \mathrm{C}$ for $2 \mathrm{~h}$ and UV-crosslinked $\left(33000 \mathrm{~mJ} / \mathrm{cm}^{2}, 30 \mathrm{~s}\right)$. Each membrane was then immersed in proteinase $\mathrm{K}(100 \mu \mathrm{g} / \mathrm{mL}$ in $2 \times \mathrm{SSC})$ for $1 \mathrm{~h}$ at $55^{\circ} \mathrm{C}$, rinsed in $2 \times \mathrm{SSC}$, washed in fresh phenylmethylsulfonyl fluoride (PMSF) $(1 \mathrm{mM}$ in $2 \times \mathrm{SSC})$ at RT for $15 \mathrm{~min}$, rinsed twice with $2 \times$ SSC and subsequently stored in a sealed bag for hybridization with one probe from the probe pool set. After hybridization and stringency washes, detection of positive hybridizations was determined, as described above.

When required for DNA sequence confirmation, PCR products (purified as described above) or plasmid minipreparations (purified using the QIA-

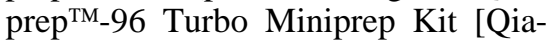
gen]) from positive hybridizations were subjected to DNA sequencing using a Model 377 or 373 DNA Sequencer with DyeDeoxy terminator chemistry (PE Biosystems). Sequences were analyzed and edited by EditView ${ }^{\mathrm{TM}}$ (PE Biosystems) or Sequencher ${ }^{\mathrm{TM}}$ (Gene Codes, Ann Arbor, MI, USA) and submitted for GenBank database comparison by NCBI BLAST programs (1).

\section{RESULTS}

To ensure rapid subtractive screening of the gridded libraries, it was nec-

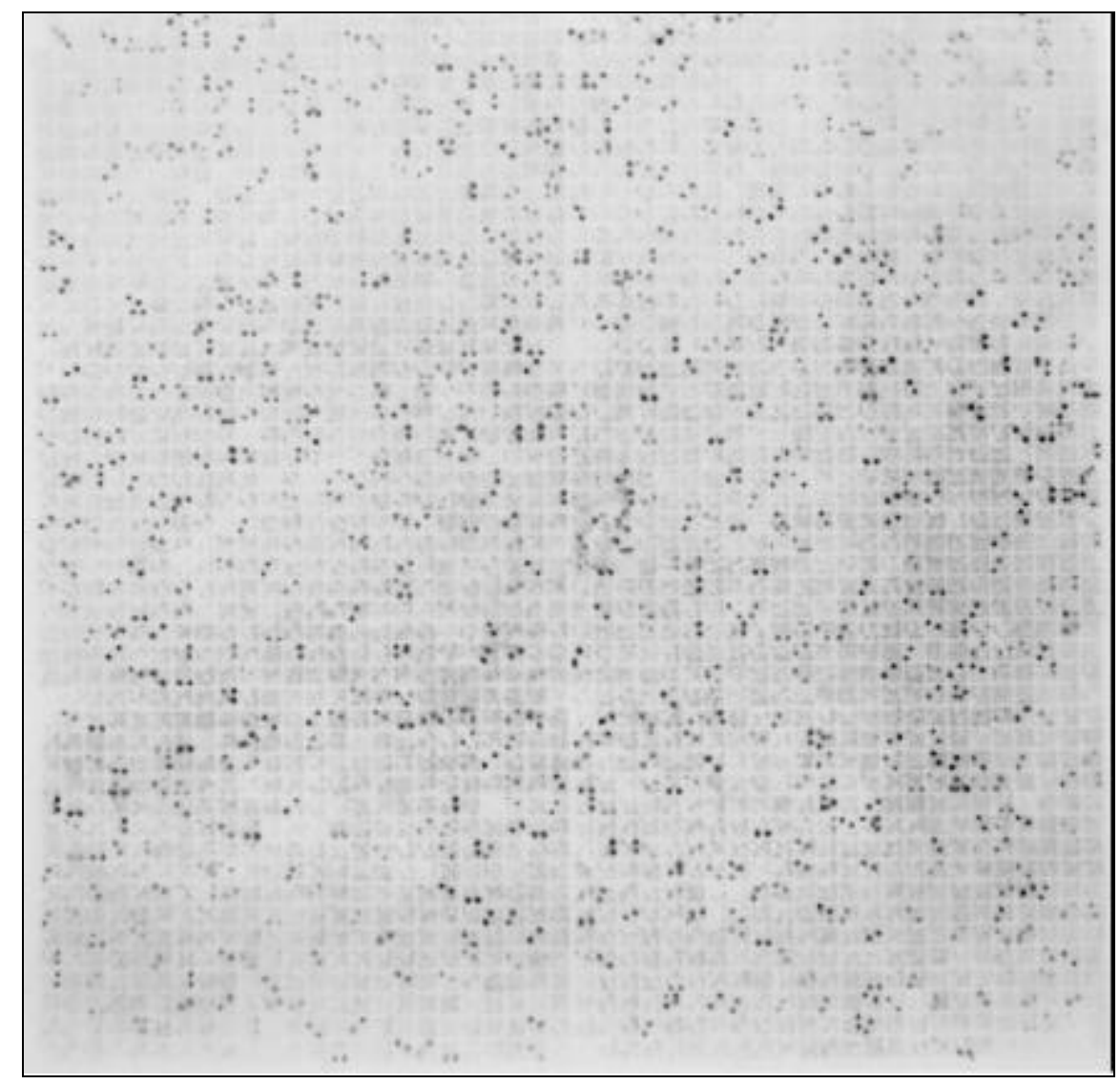

Figure 1. A high-density filter containing 18000 EST clones from a B. malayi Mf cDNA library, hybridized with a pool of 11 EST clones labeled by random priming with the NEBlot Phototope Kit and detected using the Phototope CDP-Star Detection Kit. This figure represents the 10th hybridization and stripping of the membrane. Each colony has been double spotted on the filter by the robot in a pattern reflecting the microtray and coordinates of each clone. Positive hybridizations reflect double spots consistent with the known gridding pattern. The filter was notched in one corner to ensure correct orientation 
essary to determine which probes should be utilized to screen the library. Based upon the current B. malayi DNA sequences in the database, redundancy profile data were generated (D. Guiliano and M. Blaxter, unpublished), providing a list of the highest-abundance cDNAs for screening (subtraction) from future sequencing. Several probe sets were generated; each probe set consisted of 5-10 cDNA probes.

Figure 1 presents a representative lumigram obtained after hybridization with a pool of $10 \mathrm{EST}$ probes to a Mf cDNA high-density filter. Because the gridding robot arrayed two copies of each clone in a pattern reflecting known microtray positions, positive hybridizations show "double spots," reflecting hybridization to that particular clone. Thus, the double-spot position reflects the microtray number and address of an original gridded clone, as the predicted pattern agrees with the expected pattern performed by the gridding robot (Genome Systems).

Seven sequential rounds of hybridizations to the Mf high-density filters were performed using the generated probe sets, as described above. Figure 1 presents the results for the 7 th pool (11 probes) used for subtractive hybridization (corresponding to the 10th time this filter was hybridized and stripped). It was observed that there is very low background and high signal-to-noise ratio. There was no crossover of signal detection between subsequent probings, showing that the stripping procedure was effective (data not presented).

Figure 1 shows that there are intensity differences among the sets of "double spots". This represents hybridization signals that are stronger for some sets than for others. It is interesting to note that the spot intensities within a pair of spots (i.e., duplicates of the same clone from a given microtray and position) are similar. The differences in intensity, however, do vary from clone to clone (among pairs of double spots), suggesting different target DNA amounts bound to the filters after processing and before hybridization. This effect is independent of the number of times the filters have been stripped and reprobed.

Each of the seven hybridizations provided specific positive signals on the filters. For each hybridization, the microtray positions of the positives were noted, and randomly selected clones were picked and grown. From the seven

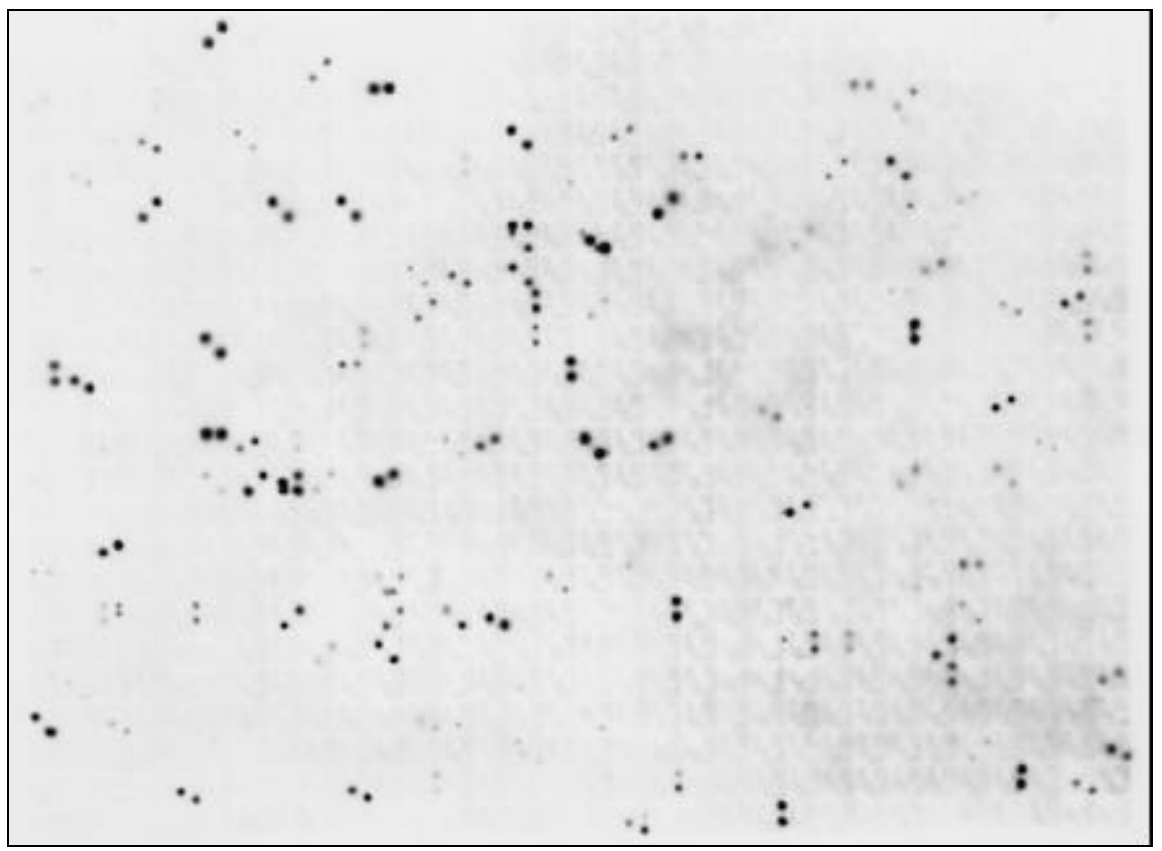

Figure 2. A high-density filter containing 5000 BAC clones from a $B$. malayi genomic library hybridized with a pool of five EST clones labeled by random priming with the NEBlot Phototope Kit and detected using the Phototope CDP-Star Detection Kit. As in Figure 1, each colony has been double spotted on the filter reflecting its original microtray location. Positive hybridizations are consistent with the known gridding pattern of the robot. The filter was notched in one corner to ensure correct orientation. 
probe sets, 100 positives were selected, plasmid minipreparations were performed and the DNA was sequenced. The resulting sequence information was submitted to GenBank database for BLAST analysis. Ninety-seven percent of the sequences represented a correct member of the appropriate probe pool set. In addition, within each probe pool, numerous representatives of the members of the pool were observed. Statistically, they were randomly distributed, based upon the abundance of each probe in the cDNA data set (Poisson distribution chi-square values $P>0.05$ within each set). The remaining 3\% positives appear to be due to cross-hybridizations; as we did not fully sequence these clones, it remains a distinct possibility that there are sequences within them that cross-hybridize to members of that particular probe set. It also is feasible that a small amount of cross-contamination among microwells occurred when sampling clones; this could account for positive hybridization, but negative sequencing results.

Based upon these results, 986 clones that had not hybridized to any of the probe sets were selected, miniprepped and DNA-sequenced. After BLAST analysis from the GenBank databases, none of these sequences $(0 \%)$ were members of any of the previously used probe sets. Sixty-one percent of these sequences reflected novel gene discovery in the Mf cDNA data set, and $43 \%$ were found to be novel to the entire Brugia cDNA project data set. Thirtynine percent of these new sequences were found to match previous cDNAs in the Mf cDNA data set and reflect cDNAs that will be useful to use to subtract in subsequent hybridization rounds, as some were found several times. The results indicate a $60 \%$ increase in novel gene discovery among seven cDNA intra-library comparisons and a 95\% increase in novel gene discovery within the Mf data set, as compared with the rate of novel gene discovery before this subtraction method was applied. Screening by chemiluminescent hybridization is highly useful in reducing redundant cDNA sequencing. With additional data, additional sets of probes can be used to perform further subtraction (in progress). The $B$. malayi $\mathrm{L} 3$ gridded library has also been subjected to this subtraction hybridization protocol. Preliminary results indicate a $100 \%$ increase in the finding of novel genes in the total Brugia data set after six rounds of subtraction hybridization (M. Ganatra and B.E. Slatko, unpublished).

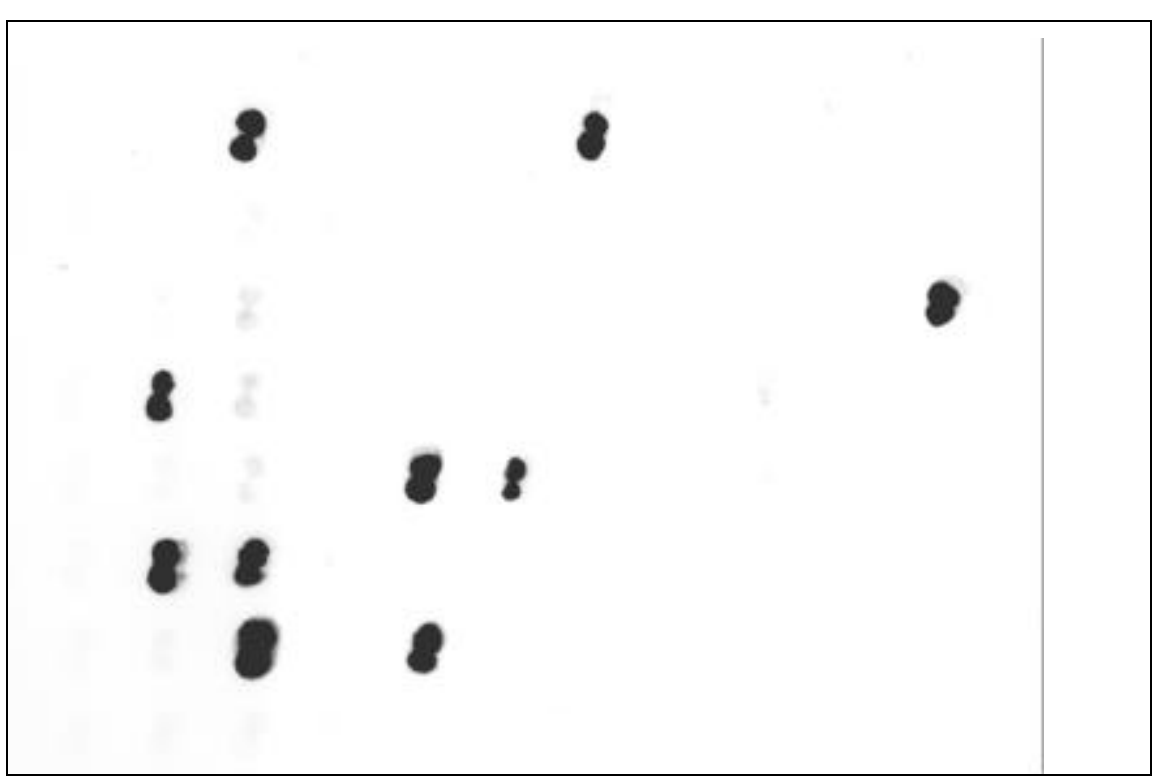

Figure 3. An example of a "deplexed" filter, hybridized with an individual probe from a probe set that was labeled by random priming with the NEBlot Phototope Kit and detected using the Phototope CDP-Star Detection Kit. Ninety-six colonies were double-spotted onto the filter, each spotted twice. Ten positive hybridizations occurred, and each shows two adjacent hybridization spots. The filter was notched on one corner to ensure correct orientation.

\section{BAC and Cosmid Filter Hybridizations}

A second use of the chemiluminescent multiplexing probe technology is to perform low-density mapping using the cDNA (EST) probes as sequence tag site (STS) markers. Along with BAC, yeast artificial chromosome (YAC) and cosmid end sequences, these will form the framework for generating overlapping $\mathrm{BAC}$, cosmid and YAC maps. Probes were made as described above, pooled in groups of 5-10 and hybridized to BAC high-density gridded arrays. From the results of the "deplexing" of the probe sets, individual BAC positions were identified for each probe. Figure 2 shows an example of a BAC filter hybridized with a probe pool set (multiplexed). As described for the EST hybridization filters, double spots reflect positive hybridization signals on clones gridded by the robot. The pattern and position of the spots identifies the microtray and position of the original clone. As with the data presented in Figure 1 for the EST filter hybridizations, the BAC hybridizations show intensity differences between sets of double spots. Since this hybridization is with a "pool" of probes, it might be suggested that differences in labeling efficiency of the individual probes before pooling for hybridization (less-labeled probes) cause weaker hybridization signals. However, no correlation exists between individual probes and spot intensities (e.g., the same probe can give darker double spots or weaker double spots). Thus, as in the case for the EST filter hybridization data, the differential hybridization signal intensities among sets of double spots likely reflects differential colony (target) DNA on the filters after processing and before hybridization. As before, this is independent of the number of times the filters have been stripped and reprobed.

A subsequent "deplexed" filter, using one probe, is shown in Figure 3. The double spots reflect an individual colony spotted twice on a filter and processed for hybridization. The multiplexing chemiluminescent method is therefore useful for detecting sequences that are present in one, or a few, copies per cell. 


\section{CONCLUSIONS}

This chemiluminescent hybridization method enables a rapid and nonradioactive approach to genomic analysis and a method of subtraction for reducing redundant sequencing. Previous work has also shown the utility of nonradioactive detection for DNA sequencing, colony hybridizations and Southern and northern blots $(8-11,17,19)$.

Multiplexing for EST mapping greatly speeds up the process of hybridization with multiple probes because the process is in parallel. An additional advantage of this approach is that the original BAC filters need not be excessively hybridized and stripped (once vs. ten times for each 10 probes), increasing the useful life of the filter.

This technique has enabled our project to proceed at a rapid pace toward the goals of both novel gene discovery by EST sequencing and long-range mapping of the Brugia genome. As with other cDNA projects, the approaches described above will allow the rapid accumulation and localization of thousands of filarial-expressed genes and the contig mapping of the Brugia genome. The hybridization results will aid in full-length gene identification, in STS and EST mapping for gene findings and in generating contigs within the cosmid and BAC libraries.

Because of close evolutionary similarity among the filarial parasites, the B. malayi initiative will provide insight into the biology of W. bancrofti and related filarial parasites such as Onchocerca volvulus. In addition, comparison to expressed genes in the distantly related free-living nematode Caenorhabditis elegans, will enable the identification of "parasite"-specific genes. This information will be important in the identification of potential novel drug and vaccine targets for these widespread parasitic diseases.

\section{ACKNOWLEDGMENTS}

We wish to thank Michelle LizotteWaniewski, Sandra Laney and Lori Saunders for providing the cDNA libraries. We also wish to thank Donald Comb, Ira Schildkraut, Richard J. Roberts and Michael Comb for support of this project. This research was funded in part by New England Biolabs, Inc. and in part by the World Health Organization/UNDP/World Bank/WHO Special Programme for Research and Training in Tropical Diseases (TDR).

\section{REFERENCES}

1.Altschul, S.F., W. Gish, W. Miller, E.W. Myers and D.J. Lipman. 1990. Basic local alignment search tool. J. Mol. Biol. 215:403410.

2.Ausubel, F.M., R. Brent, R.E. Kingston, D.D. Moore, J.G. Seidman, J.A. Smith and K. Struhl. 1989. Current Protocols in Molecular Biology. Wiley-Interscience, New York.

3.Birren, B., E.D. Green, S. Klapholz, R. Myers and J. Roslams. 1997. Genome Analysis: A Laboratory Manual. CSH Laboratory Press. Cold Spring Harbor, NY.

4.Blaxter, M.L. 1995. The Filarial Genome Project. Parasitol. Today 11:811-812.

5.Blaxter, M.L. and M. Aslett. 1997. Internet resources for the parasite genome projects. Trends Genet. 13:40-41.

6.Blaxter, M.L., J. Daub, M. Waterfall, D. Guiliano, S. Williams, K. Jayaraman, R. Ramzy, B. Slatko and A.L. Scott. 1998. The
Filarial Genome Project. In COST 819. The Commission of the European Community, Brussels. p. 47-58.

7.Blaxter, M.L., N. Raghavan, I. Ghosh, D. Guiliano, W. Lu, S.A. Williams, B. Slatko and A.L. Scott. 1996. Genes expressed in Brugia malayi infective third stage larvae. Mol. Biochem. Parasitol. 77:77-93.

8.Creasey, A., L. D'Angio, T.S. Dunne, C. Kissinger, T. O'Keefe, H. Perry-O'Keefe, L. Moran, M. Roskey, I. Schildkraut, L.E. Sears and B. Slatko. 1991. Application of a novel chemiluminescence-based DNA detection method to single-vector and multiplex DNA sequencing. BioTechniques 11:102-109.

9.Dubitsky, A., J. Brown and H. Brandwein. 1992. Chemiluminescent detection of DNA on nylon membranes. BioTechniques 13:392400.

10.Kern, S. and G.M. Hampton. 1997. Direct hybridization of large-insert genomic clones on high-density gridded filter arrays. BioTechniques 23:120-124.

11.Kincaid, R.L. and M.S. Nightingale. 1988. A rapid non-radioactive procedure for plaque hybridization using biotinylated probes prepared by random primed labeling. BioTechniques 6:42-49.

12.New England Biolabs. 1997. Phototope ${ }^{\circledR}$ Star Detection Manual. Beverly, MA.

13.Ottesen, E.A. and C.D. Ramachandran. 1995. Lymphatic filariasis. Infection and disease: control strategies. Parasitol. Today 11:129-131.

14.Pomrehn, A. 1997. The construction of a Brugia malayi cosmid library for analysis of $B$. malayi genome organization. Northhampton, MA: Smith College; Thesis.

15.Pope-Chappell, J. 1997. Construction and characterization of a genomic Brugia malayi library using bacterial artificial chromosomes. Northhampton, MA: Smith College; Thesis.

16.Sambrook, J., E.F. Fritsch and T. Maniatis. 1989. Molecular Cloning: A Laboratory Manual, 2nd ed. CSH Laboratory Press, Cold Spring Harbor, NY.

17.Sheffield, J.S., W.H. Benjamin, Jr. and L.S. McDaniel. 1992. Detection of DNA in Southern blots by chemiluminescence is a sensitive and rapid technique. BioTechniques 12:836839.

18.Unasch, T.R. 1994. The filarial genome project. Parasitol. Today 10:415-416.

19.Weintraub, D., P. Ulanch and J. Mullet. 1998. Screening BAC high density filters with Southern-Star ${ }^{\mathrm{TM}}$. Luminescence Topics 6:3-4.

Received 15 January 1999; accepted 18 March 1999.

\section{Address correspondence to:}

Dr. Barton Slatko

Molecular Parasitology Group

New England Biolabs, Inc.

32 Tozer Road

Beverly, MA 01915, USA

Internet:dnaseq@neb.com 